\title{
ESTABLECIMIENTO DEL Lolium multiflorum CON CINCO DENSIDADES SOBRE PASTURAS DEGRADADAS COMO UNA ALTERNATIVA A LA SIEMBRA DE CULTIVOS AGRÍCOLAS
}

\author{
José Ordóñez F. ${ }^{1,2}$ y Custodio Bojórquez R. ${ }^{1}$
}

\section{Abstract}

\begin{abstract}
The present study had the objective to evaluate the establishment of Italian Rye grass (Lolium multiflorum) under five densities of seeding (15, 30, 45, 60 and $75 \mathrm{~kg}$ of $\mathrm{seed} / \mathrm{ha}$ ) into degraded pastures invaded by kikuyu (Pennisetum clandestinum), as an alternative to rotations with agricultural cultivations. Each density was mixed with $3.0 \mathrm{~kg}$ / ha of Lucerne seed (Ranger variety). The study was carried out at the "El MantaroIVITA" Research Station from March till July 2002. A land with degraded pasture that was grazed for seven years was mechanically prepared and the Italian Rye grass seed was vollied by hand. The fertilization was carried out with $60-20-0$ of $\mathrm{N}-\mathrm{P}_{2} \mathrm{O}_{5}-\mathrm{K}_{2} \mathrm{O} \mathrm{kg} / \mathrm{ha}$ thirty days after seedding. The statistical analysis was done using the randomized complete block design with three repetitions. The botanical composition before the first grazing (120 days after seeding) showed a significant increase $(\mathrm{p}<0.05)$ of the Italian Rye grass proportion ( 21.3 to $82.9 \%$ with the seeding density of 15 and $75 \mathrm{~kg}$, respectively). The biomass production of Italian Rye grass significantly increased $(\mathrm{p}<0.05)$ with the increase of seeding density. It was concluded that the Italian Rye grass is better established into degraded areas invaded by kikuyu, when the seeding density was $>30 \mathrm{~kg} / \mathrm{ha}$.
\end{abstract}

Key words: establishment, density of seeding, degraded pastures, Italian Rye grass, kikuyu

\section{Resumen}

El objetivo del estudio fue determinar el establecimiento del Rye grass italiano "Magnum" con cinco densidades de siembra sobre terrenos con pastos degradados e invadidos por kikuyu (Pennisetum clandestinum) como una alternativa a la rotación con cultivos agrícolas. El estudio se realizó en el Centro de Investigaciones IVITA-El Mantaro (Junín) entre marzo y julio del 2002. Se sembró al voleo 15, 30, 45, 60 y $75 \mathrm{~kg}$ de semilla/ha de Lolium multiflorum (Rye grass italiano) en un suelo con pastura degradada de siete años de pastoreo y preparado mecánicamente. Cada densidad se mezcló con $3.0 \mathrm{~kg} / \mathrm{ha} \mathrm{de}$ semilla de alfalfa "Ranger". Se fertilizó con 60-20-0 Kg/ha de $\mathrm{N}-\mathrm{P}_{2} \mathrm{O}_{5}-\mathrm{K}_{2} \mathrm{O}$ treinta días después de la siembra y se utilizó el diseño de block completo randomizado con tres repeticiones. La composición botánica a los 120 días de la siembra (antes del primer pastoreo) indicó que la proporción del Rye grass italiano se incrementó en forma signifi-

\footnotetext{
${ }^{1}$ Estación Experimental del Centro de Investigaciones IVITA - El Mantaro, FMV-UNMSM

${ }^{2}$ E-mail: hugor_48@hotmail.com
} 
cativa con las densidades de siembra $(\mathrm{p}<0.05)$. Las producciones de materia verde del Rye grass italiano se incrementaron en forma significativa $(\mathrm{p}<0.05)$ con el aumento de la densidad de siembra (21.3 a $82.9 \%$ con densidades de siembra de 15 y $75 \mathrm{~kg} / \mathrm{ha}$, respectivamente). Se concluye que el Rye grass italiano se establece en áreas degradadas invadidas por el kikuyu cuando se siembra con densidades mayores a $30 \mathrm{~kg} / \mathrm{ha}$ de semilla total.

Palabras clave: establecimiento, densidad de siembra, pastura degradada, Rye grass italiano, kikuyu

\section{INTRODUCCIÓN}

La mejor densidad de siembra en pasturas es aquella que permite una cobertura rápida del suelo (Walton, 1983). En el Valle del Mantaro, el productor utiliza $30 \mathrm{~kg} / \mathrm{ha}$ de semilla total (gramíneas + leguminosas) en suelos con manejo agrícola anterior. Sin embargo, poco se sabe sobre la siembra directa en áreas degradadas, pero se espera que mayores densidades a las recomendadas puedan servir para eliminar o controlar especies nodedas (O roóñez et al., 2003; Ordóñez y Bojórquez, 2001b). La degradación de los pastos es atribuible a la compactación por pérdida de fertilidad de suelos, así como a la invasión de kikuyu (Pennisetum clandestinum), gramínea naturalizada que invade los terrenos a través de semilla vegetativa y sexual.

Actualmente, para sembrar pasturas en áreas degradadas, se debe mecanizar el suelo y sembrar papa, maíz y habas por tres años. De esa forma, los deshierbos sucesivos van eliminando las plántulas del kikuyu provenientes de semilla acumulada en muchos años. (Bojórquez, 1992), y cuando el suelo está limpio se procede a la siembra de especies forrajeras. Sin embargo, el ganadero requiere de forraje en forma constante y poco le interesa la siembra de cultivos. No existe una tecnología de siembra de pasturas sobre áreas con pastos degradados, de allí que el objetivo del presente estudio fue realizar el establecimiento de Rye grass italiano con cinco densidades de siembra sobre pastos degradados (invadidos por kikuyu) como una alternativa a la rotación con cultivos agrícolas (Bojórquez, 1998).

\section{Matteriales y Métodos}

El estudio se realizó entre marzo y julio del 2002 en el Instituto Veterinario de Investigaciones Tropicales y de Altura (IVITA - El Mantaro), ubicado en el departamento de Junín, provincia de Jauja. La zona tiene una altitud de 3,320 msnm, una temperatura promedio anual de $11^{\circ} \mathrm{C}$ y una precipitación promedio anual de $770 \mathrm{~mm}$.

En una pastura degradada ( $L$. multiflorum, L. perenne, D. glomerata, Trifolium repens y $T$. pratense) de siete años de uso, se preparó el suelo con arado de discos, pase cruzado de rastra, nivelado y levantamiento de camellones, logrando melgas de $3 \mathrm{~m}$ de ancho. Se sembraron 15, 30, 45, 60 y $75 \mathrm{~kg}$ de semilla/ha de Rye grass italiano, cada una mezclada con $3 \mathrm{~kg}$ de semilla/ha de alfalfa "Ranger". Se fertilizó con 60-20-0 kg de $\mathrm{N}-\mathrm{P}_{2} \mathrm{O}_{5}-\mathrm{K}_{2} \mathrm{O}$ por hectárea, treinta días después de la siembra.

Se evaluó el establecimiento de la pastura y la biomasa verde utilizando el diseño de Block Completo Randomizado con tres repeticiones. Para la composición botánica se utilizó el método de rango de peso seco (Mannetje y Haydock, 1980) y para la disponibilidad de materia seca el método de rendimientos comparativos (Haydock y Shaw, 1975). 


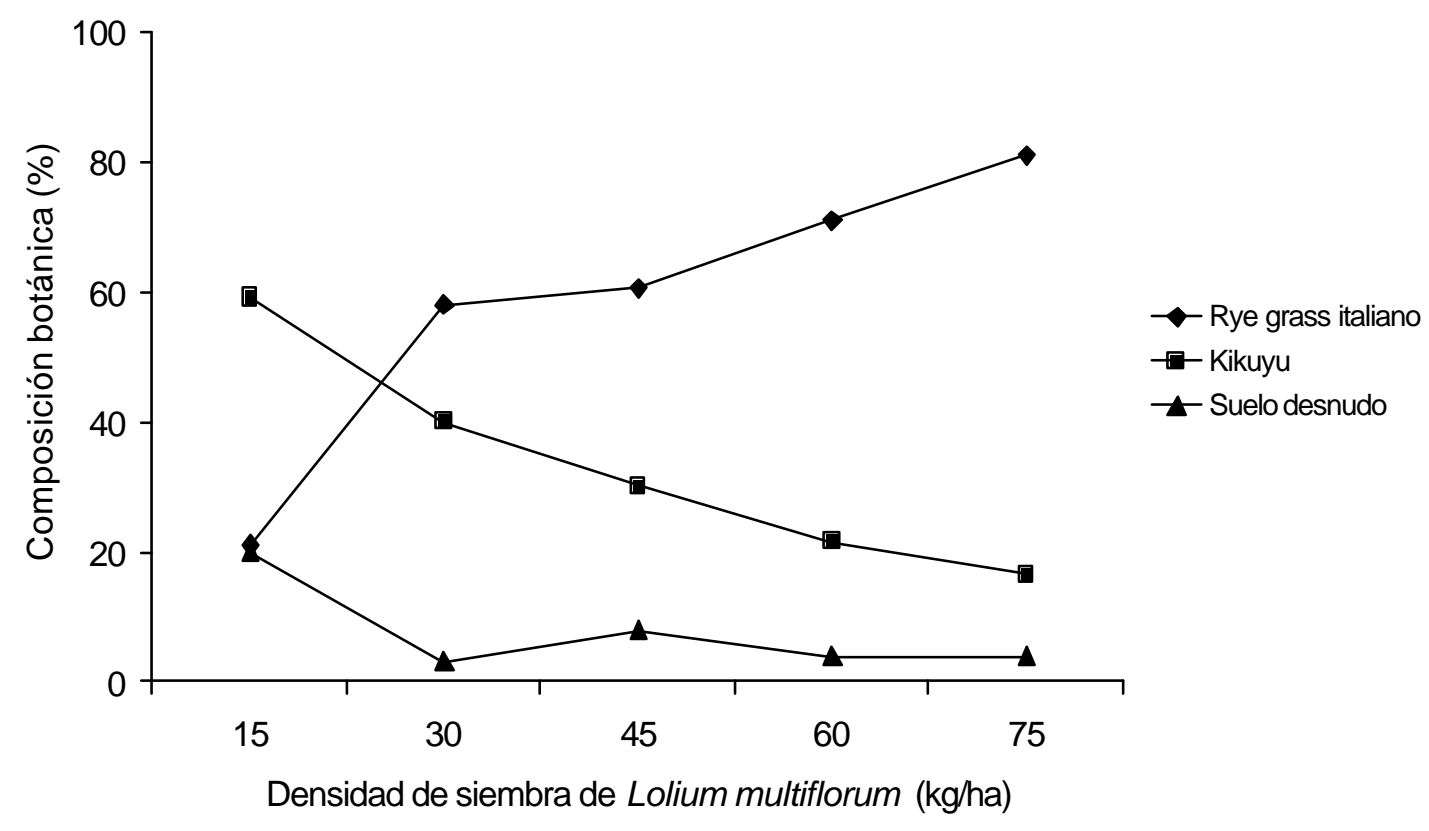

Figura 1. Composición botánica 120 días después de la siembra del Lolium multiflorum en terrenos con pasturas degradadas en el valle del Mantaro

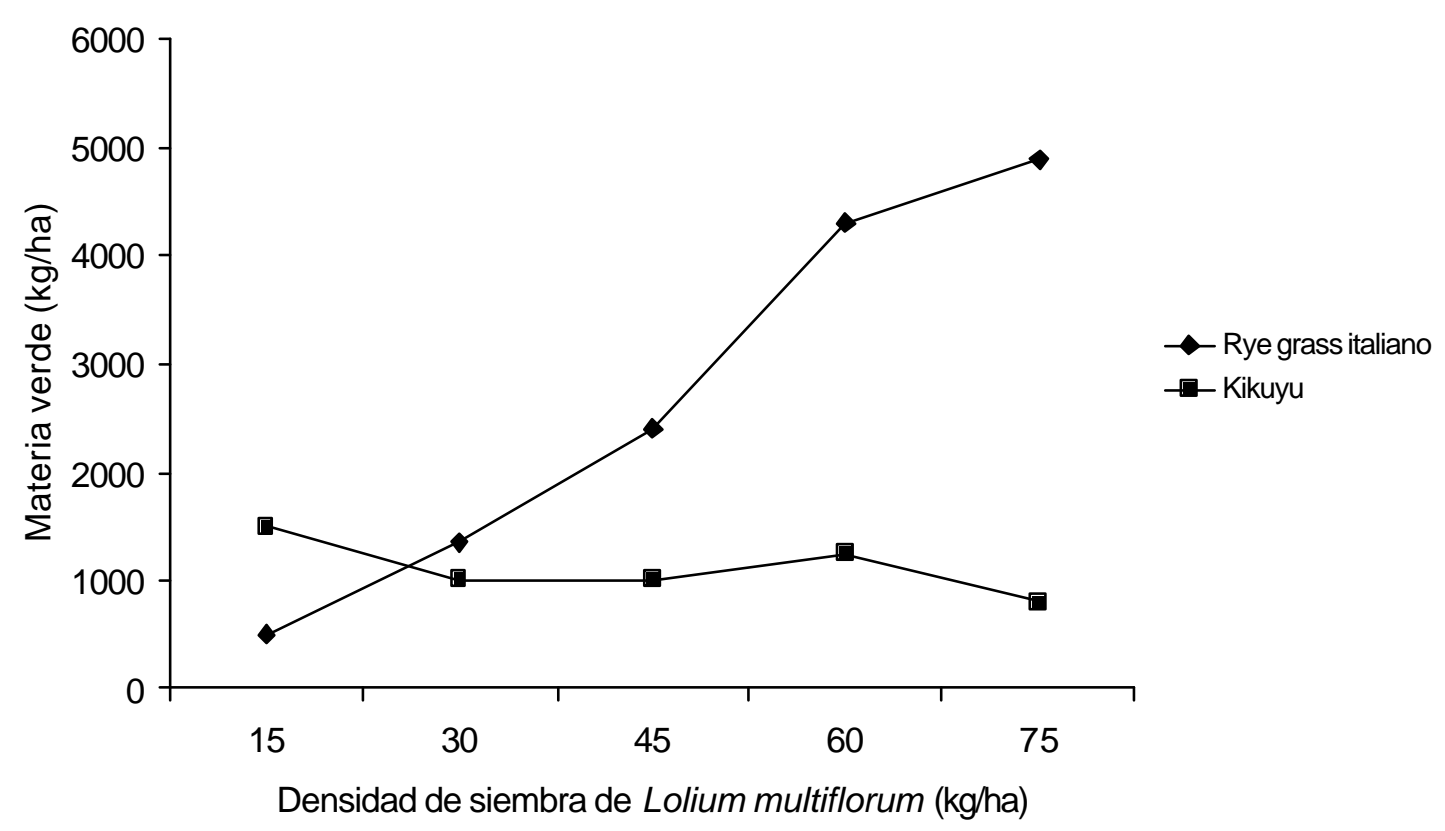

Figura 2. Producción de materia verde (TM/ha) a los 120 días de la siembra del L. multiflorum en terrenos con pasturas degradadas en el valle del Mantaro 


\section{Resultados y Discusión}

La pastura se sembró y evaluó en ausencia de lluvias (abril-julio) en un sistema bajo riego. La composición botánica se limitó al Rye grass italiano y al kikuyu. Esta población es diferente cuando se siembra pastura después de un cultivo, donde la variabilidad de maleza es amplia, con predominio de especies de hoja ancha (Ordóñez y Bojórquez, 2001a).

La composición botánica a los 120 días después de la siembra (antes del primer pastoreo) mostró un incremento significativo $(\mathrm{p}<0.05)$ de Rye grass italiano por efecto de las densidades de siembra $(21.3 \%$ con 15 $\mathrm{kg}$ de semilla/ha y de $82.9 \%$ con $75 \mathrm{~kg}$ de semilla/ha; Fig. 1). Estos resultados muestran su adaptación al valle del Mantaro dado su rápido crecimiento, vigor inicial y competencia por nutrientes con el kikuyu. Aparentemente el Rye grass italiano con $75 \mathrm{~kg} / \mathrm{ha}$ disminuyó la presencia del kikuyu (12.3\%).

La leguminosa sembrada no presentó el vigor suficiente para ser detectada a los 120 días de la siembra (Fig. 2). Las biomasas de Rye grass italiano, según la densidad de siembra, fueron significativamente diferentes $(\mathrm{p}<0.05)$. El Rye grass italiano produjo 556 $\mathrm{kg} / \mathrm{ha}$ de materia verde con la menor densidad de siembra $(15 \mathrm{~kg} / \mathrm{ha})$ y $4,831 \mathrm{~kg} / \mathrm{ha}$ con la mayor densidad (75 kg/ha), indicando que las altas densidades de siembra tienen mayores oportunidades de competir con el kikuyu. Se observó que el sistema radicular de las densidades altas son profusas, logrando disminuir las actividades fisiológicas del kikuyu, traducido en una pobre producción de materia verde $(714 \mathrm{~kg} / \mathrm{ha}$ en la mayor densidad de siembra (Fig. 2).

El Rye grass italiano y el kikuyu tienen básicamente los mismos requerimientos para su normal crecimiento y desarrollo. Ellos requieren y compiten por un adecuado suministro de agua, nutrientes, luz, temperatura, dióxido de carbono y espacio para crecer. En una comunidad de plantas, la especie más agresiva usualmente domina. Esta agresividad está asociada con un crecimiento rápido que produce sombra a las demás plantas, impidiendo su acceso a la luz solar. También tienen un eficiente sistema radicular para absorber y utilizar los nutrientes y la humedad del suelo (Anderson, 1983).

\section{ConClusiones}

El Rye grass italiano se puede establecer en áreas degradadas invadidas por el kikuyu cuando se siembra con densidades mayores a $30 \mathrm{~kg} / \mathrm{ha}$ de semilla total. Sin embargo, la dinámica de la composición botánica y producción forrajera en el tiempo debe ser evaluada debido a la agresividad y persistencia del kikuyu.

\section{Literatura Citada}

1. Anderson, W.O. 1983. Weed science. Principles. West publishing. USA. $34 \mathrm{p}$.

2. Bojórquez, R.C. 1992. El IVITA y la producción lechera del valle del Mantaro. Alma Mater UNMSM 3: 71-76.

3. Bojórquez, R.C. 1998. Pastos y fuentes de alimentos para el ganado en la sierra central del Perú. ILEIA/GIAREC. Huancayo.

4. Haydock, K.P.; N.H. Shaw. 1975. The comparative yield method for estimating dry matter yield of pasture. Austr. J. Exp. Agric. Anim. Husb. 15: 663-670.

5. Mannetje, L.T.; J.P. Haydock. 1980. The dry weight rank method for the botanical analysis of pasture. J. British Grassland. Soc. 18: 268-275.

6. Ordóñez, J.H.; C. Bojórquez. 2001a. Evaluación comparativa del establecimiento de pasturas cultivadas en el valle del Mantaro. Resúmenes XXIV Reunión Científica Anual Peruana de Producción Animal. Lima. p 238. 
7. Ordóñez, J.; C. Bojórquez. $2001 b$. Efecto de la densidad de siembra del Trifolium repens en la composición botánica y producción de una pastura asociada. Resúmenes XXIV Reunión Científica Anual de la Asociación Peruana de Producción Animal. Lima. p 235.

8. Ordóñez, J.; C. Bojórquez; C. Noli. 2003. Manejo de la densidad y cubri- miento de la semilla en el establecimiento y producción de pasturas cultivadas. Resúmenes XXVI Reunión Científica Anual de la Asociación Peruana de Producción Animal. Pucallpa. p 161.

9. Walton, P.D. 1983. The production and management of cultivated forages. Reston. Virginia, USA. 